\title{
Technè
}

La science au service de l'histoire de l'art et de la préservation des biens culturels

46 | 2018

Science et conservation

\section{L'œuvre de Frédéric Bazille (1841-1870) examiné au laboratoire}

The works of Frédéric Bazille (1841-1870) examined in the laboratory

\section{Bruno Mottin}

\section{OpenEdition}

Journals

Édition électronique

URL : http://journals.openedition.org/techne/335

DOI : $10.4000 /$ techne.335

ISSN : 2534-5168

Éditeur

C2RMF

Édition imprimée

Date de publication : 1 décembre 2018

Pagination : $30-38$

ISBN : 978-2-11-152829-1

ISSN : $1254-7867$

Référence électronique

Bruno Mottin, «L'œuvre de Frédéric Bazille (1841-1870) examiné au laboratoire », Technè [En ligne], 46 | 2018, mis en ligne le 19 décembre 2019, consulté le 22 juillet 2020. URL : http:// journals.openedition.org/techne/335; DOI : https://doi.org/10.4000/techne.335

La revue Technè. La science au service de l'histoire de l'art et de la préservation des biens culturels est mise à disposition selon les termes de la Licence Creative Commons Attribution - Pas d'Utilisation Commerciale - Pas de Modification 4.0 International. 


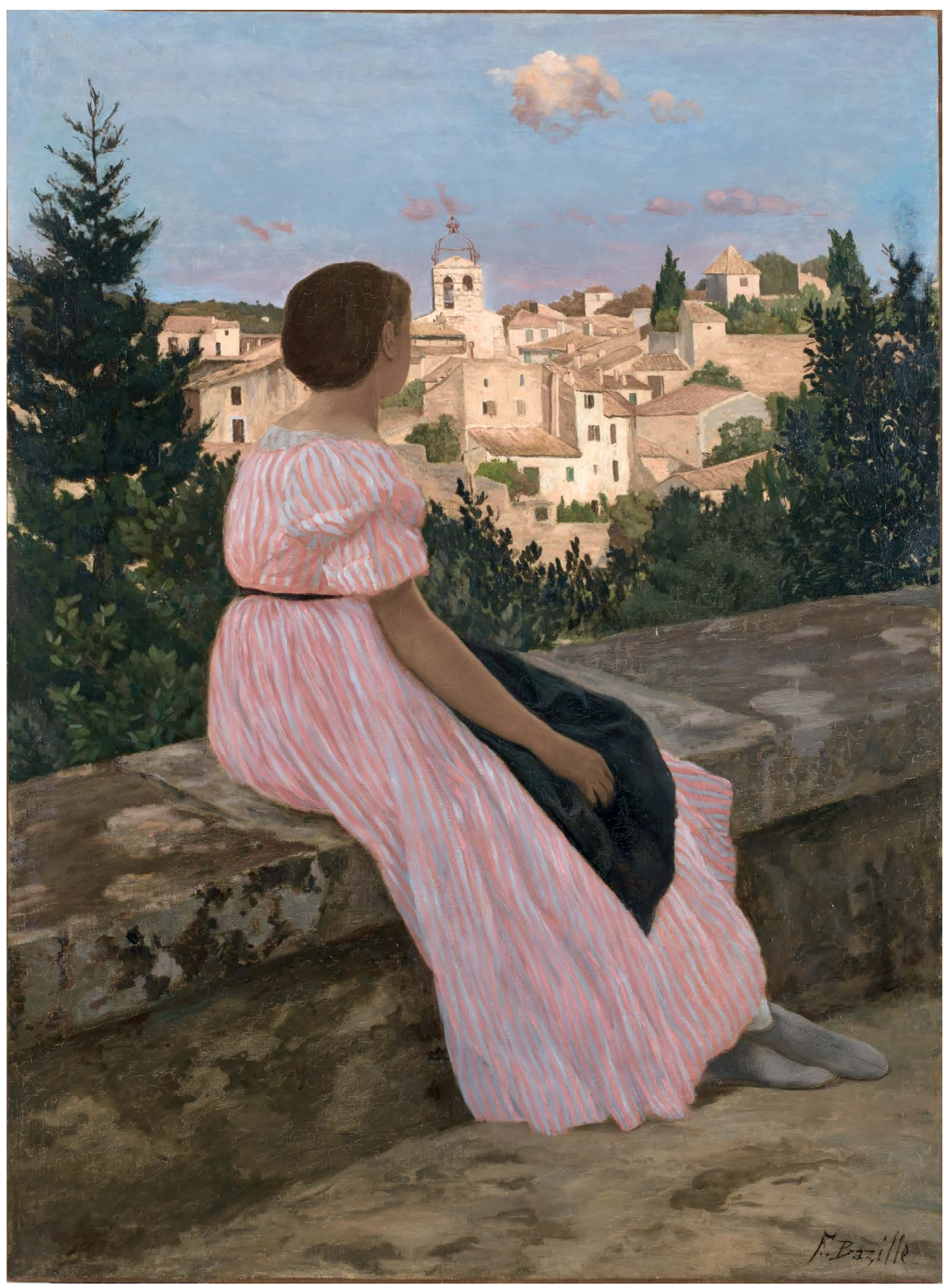

Fig. 1. Frédéric Bazille, La Robe rose, 1864-1865 ou après, huile sur toile (147,5 x 109,5 cm),

Paris, musée d'Orsay (inv. RF 2450). Après nettoyage, par B. Trémolières, 2016. (c) C2RMF/T. Clot. 


\section{L'œuvre de Frédéric Bazille (1841-1870) examiné au laboratoire}

The works of Frédéric Bazille (1841-1870) examined in the laboratory

Résumé. Une étude de laboratoire a été menée sur quatorze peintures de Bazille et de son entourage. Ce travail a permis de préciser les phases d'exécution de plusieurs cuvres, d'identifier des repentirs, de retrouver des compositions sous-jacentes et de clarifier les cas de collaboration entre Bazille et ses camarades d'atelier.

Mots-clés. Frédéric Bazille, impressionnisme, imagerie scientifique, radiographies, Paris, musée d'Orsay, Montpellier, musée Fabre.
Abstract. A laboratory study was conducted on fourteen paintings by Bazille and his circle. This research enabled us to learn more about the different phases of execution of several works, to identify the pentimenti, discover underlying compositions and clarify the cases where Bazille collaborated with his fellow artists in the studio.

Keywords. Frédéric Bazille, Impressionism, scientific imaging, X-rays, Musée d'Orsay, Paris, Musée Fabre, Montpellier.
L'exposition Frédéric Bazille, la jeunesse de l’impressionnisme, qui s'est tenue à Montpellier, Paris et Washington en 2016-2017, a montré combien cet artiste souvent négligé a joué un rôle de premier plan dans la naissance de l'impressionnisme. Admirateur précoce d'Édouard Manet, qu'il rencontre chez son cousin Lejosne peu après son arrivée à Paris, à l'automne 1862, il est l'ami, le compagnon d'atelier et souvent le soutien financier de Claude Monet, d'Alfred Sisley et d'Auguste Renoir. Il partage avec eux la même passion pour une peinture claire, vigoureuse et vibrante, qu'il enrichit de son extrême sensibilité et de la luminosité de son Languedoc natal. L'art de Bazille aurait été davantage reconnu si le peintre n'était mort sous les balles des troupes prussiennes avant d'avoir atteint 29 ans. On reste donc confondu devant la qualité et le caractère novateur de ses quelque soixante peintures, réalisées en moins de huit ans.

Lors de la préparation de cette exposition, les conservateurs du musée d'Orsay et du musée Fabre de Montpellier ont sollicité le Centre de Recherche et de Restauration des Musées de France (C2RMF) pour réaliser des études techniques sur quatorze œuvres du peintre et de ses compagnons ${ }^{1}$. Les délais et l'architecture du catalogue d'exposition n'ont malheureusement pas permis d'intégrer tous les résultats obtenus. Le présent texte voudrait pallier ce manque en présentant de façon synthétique les principales observations issues de ces examens et en proposant d'amender le catalogue sur quelques points.

\section{La Robe rose}

Le tableau intitulé La Robe rose (fig. 1) représente une jeune femme vêtue d'une robe rose rayée de gris, qui détourne la tête pour regarder le village de Castelnau-le-Lez à l'arrièreplan. Cette œuvre à la mise en page hardie et au chromatisme subtil est considérée comme l'une des toutes premières créations de Bazille, entreprise à l'été ou l'automne 1864 dans la propriété familiale de Méric, près de Montpellier, où le jeune homme est venu se reposer.

La composition est préparée par un dessin au crayon, de grand format ${ }^{2}$, qui a été mis au carreau pour être agrandi sur toile. Le dessin présente toutefois d'importantes variantes par rapport au tableau définitif car la jeune fille n'est pas représentée de dos, mais de trois quarts, est vêtue d'une ample robe, les bras posés sur le giron et le visage légèrement tourné vers la droite. Des changements de composition ont donc été opérés entre le dessin et la peinture.

La réalisation de cette ouvre n'est pas allée sans obstacles. Grâce à une lettre de Gaston Bazille adressée à son fils le 16 décembre 1864, on sait que le peintre a laissé son tableau dans la maison familiale de Méric au moment de son retour à Paris et que son père a pris l'initiative de le faire photographier par un peintre local, Huguet-Moline. Gaston Bazille doit avouer dans ce courrier qu'Huguet-Moline, « trouvant que sa photographie ne ressortait pas assez, a pris sur lui sans rien dire à personne, de passer de la couleur sur les parties écaillées de ta figure. J'ai été très vexé quand je me suis aperçu de ces retouches, malheureusement le mal était

Bruno Mottin, conservateur du patrimoine, département Recherche, C2RMF (bruno.mottin@culture.gouv.fr). 


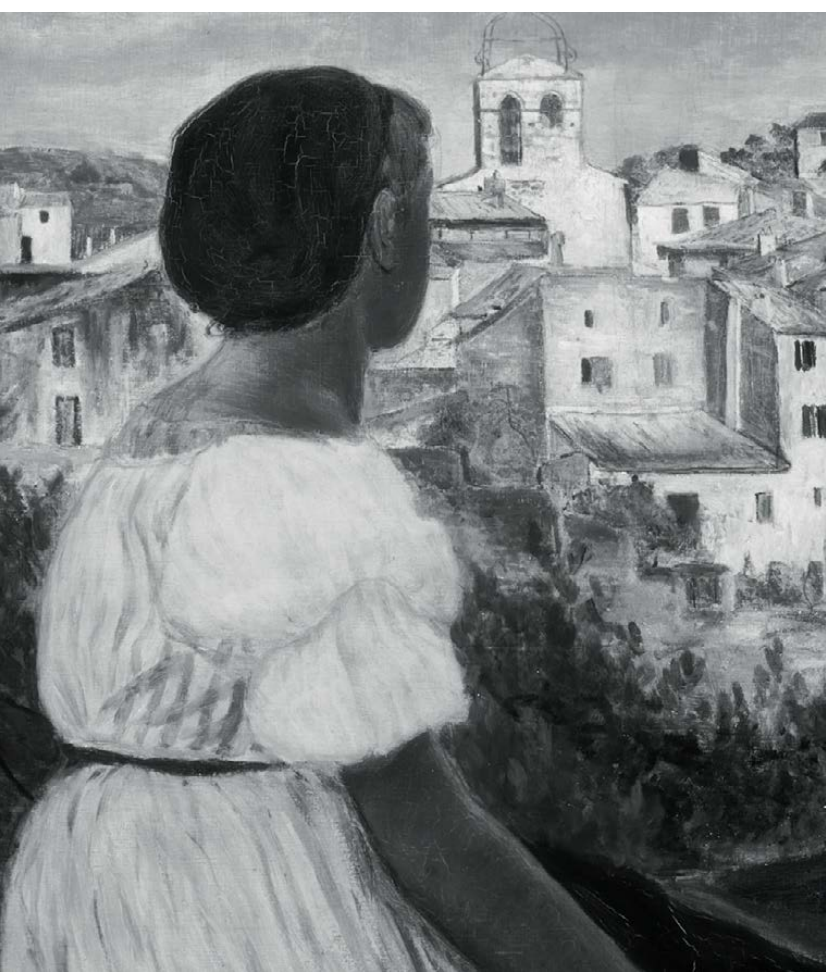

Fig. 2. La Robe rose, détail de la réflectographie infrarouge. (c) C2RMF/J.-L. Bellec.

fait. Le bras et la joue de Thérèse n'ont pas gagné au changement, tu arrangeras cela comme tu l'entendras si tu conserves cette étude $^{3}$ ». Le peintre reçoit la photographie incriminée au début de l'année 1865 et remarque que " cet animal de Moline a bien tripoté mon tableau, mais j'en fais d'autres pour le remplacer ${ }^{4} »$.

Les lettres indiquent donc que l'œuvre a été commencée en 1864, que la couche peinte a formé des craquelures prématurées qui sont appelées ici des écailles, et que l'œuvre est inachevée car elle est décrite comme une étude, que le peintre pourra arranger s'il souhaite la conserver.

L'œuvre de dimensions moyennes (147,5 x 109,5 cm) est peinte sur une toile d'armure simple à tissage serré. Elle est rentoilée, mais est encore tendue sur son châssis d'origine à croix centrale, clefs et montants d'assez faible largeur (environ $6 \mathrm{~cm}$ ). La préparation de couleur blanche ne présente aucune irrégularité de surface ; elle a probablement été posée industriellement. Au-dessus, le peintre a esquissé ses figures avec un pinceau chargé de pigments brun-rouge mélangés à de l'essence de térébenthine et parfois à un peu d'huile pour obtenir une matière fluide et transparente que l'on appelle communément la «sauce » et que l'on emploie tout au long du XIX ${ }^{\mathrm{e}}$ siècle pour ébaucher une composition ${ }^{5}$. Aucune trace de mise au carreau n’apparaît sur le réflectogramme

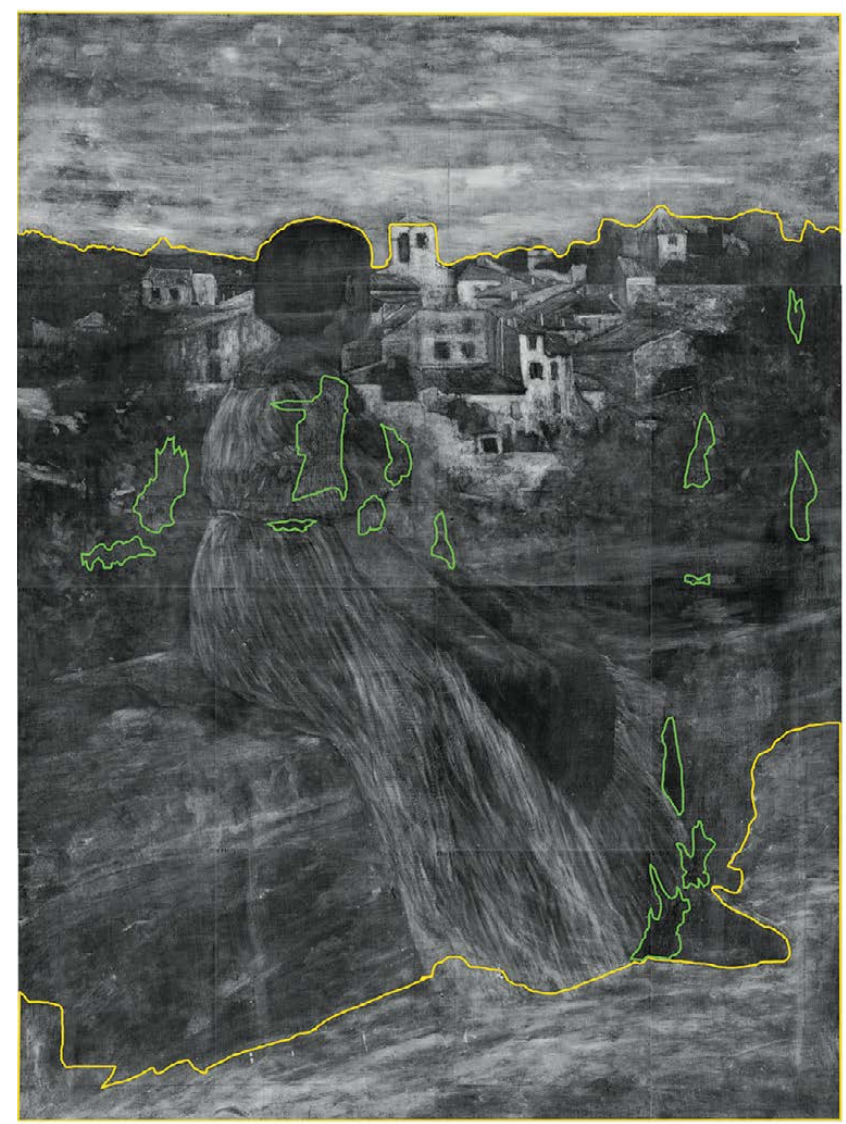

Fig. 3. La Robe rose, radiographie. En vert, traces de grattage ou d'usage de solvant; en jaune, emplacement d'une seconde couche de préparation. (C C2RMF/M. de Drée, numérisation et B. Mottin, schéma.

infrarouge (fig. 2) où l'on remarque toutefois que l'artiste a dessiné au crayon noir la structure du village et le contour des arbres de façon rigoureusement identique à son dessin.

D’après l'observation sous binoculaire, Bazille a mis en couleurs son ébauche au moyen de sous-couches faiblement modelées. Il a mis en place le village avec un ton jaune clair et peint un ciel qui était, d'après la radiographie, davantage chargé de nuages que le ciel presque pur de la composition finale (fig. 3). En bas à gauche de la radiographie, un liséré clair suggère que la robe était plus ample et l'on distingue près du torse les traces de deux bras en parties effacés. Cette composition est donc proche du dessin préparatoire. La réflectographie infrarouge confirme la présence des deux bras, mais ne permet pas de préciser si le visage était placé de trois quarts, comme sur le dessin, ou en profil perdu, comme actuellement.

De nombreux indices montrent que l'œuvre a été entièrement reprise par Bazille après sa première phase d'exécution. On observe en effet des traces de raclage au couteau à l'emplacement de la figure qui montrent que l'artiste a gratté des surépaisseurs de matière près des bras, dans la végétation et sur les pieds. Ailleurs, la présence de bulles et de grumeaux à la surface de la préparation fait supposer que Bazille a employé des solvants pour dissoudre la couche colorée. Enfin, 
Fig. 5. Frédéric Bazille, La Réunion de famille, 1867 , huile sur toile

$(152 \times 230 \mathrm{~cm})$, Paris,

musée d'Orsay (inv.

RF 2749) : radiographie.

En jaune, mise en évidence

de la première composition.

(C) C2RMF/J. Marsac,

numérisation et B. Mottin, schéma.
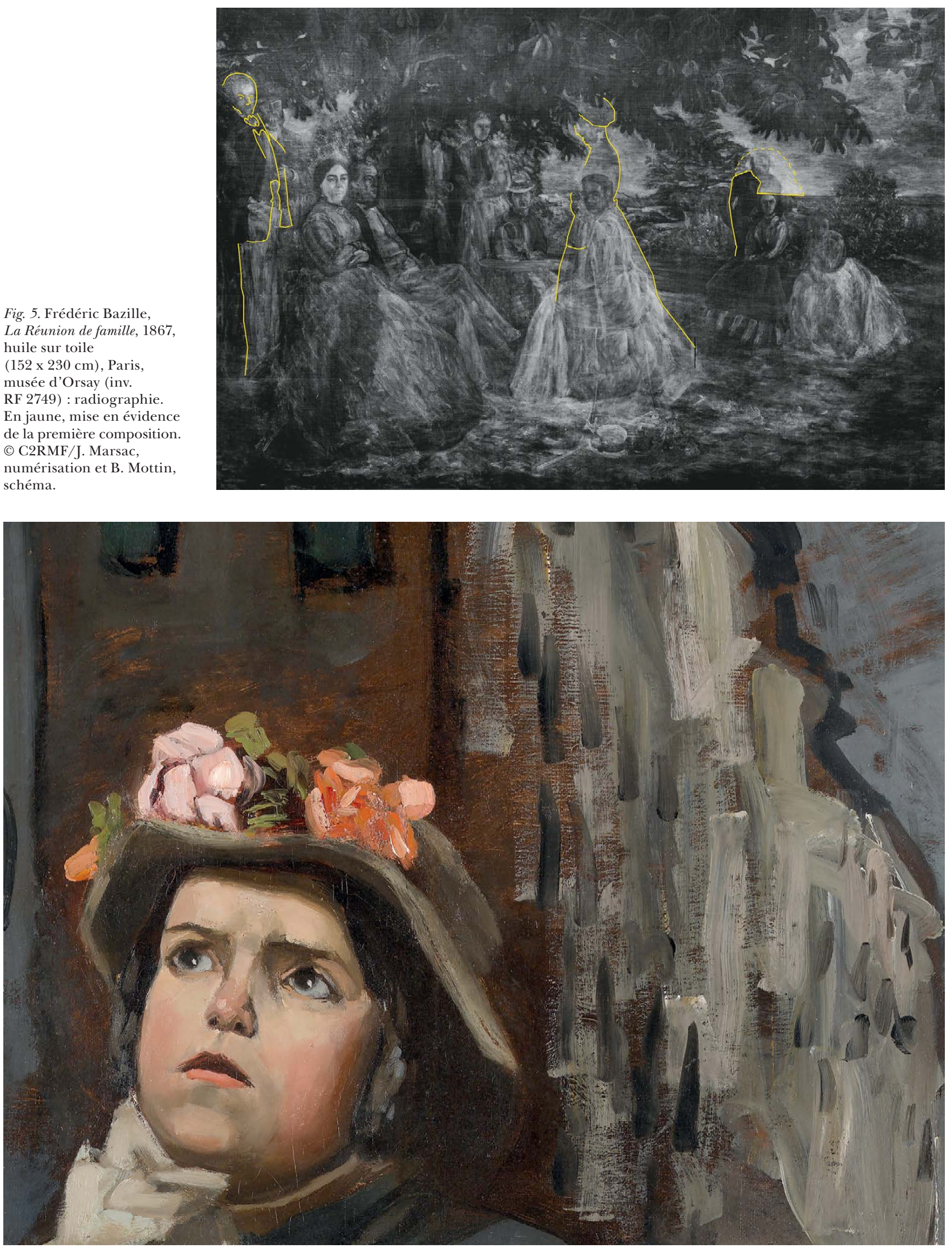

Fig. 4. Frédéric Bazille, Petite Italienne chanteuse des rues, 1866, huile sur toile $(130,5 \times 97,5 \mathrm{~cm})$,

Montpellier, musée Fabre (inv. 2002.5.1) : détail de la tête et des immeubles. (C C2RMF/G. Parisse. 


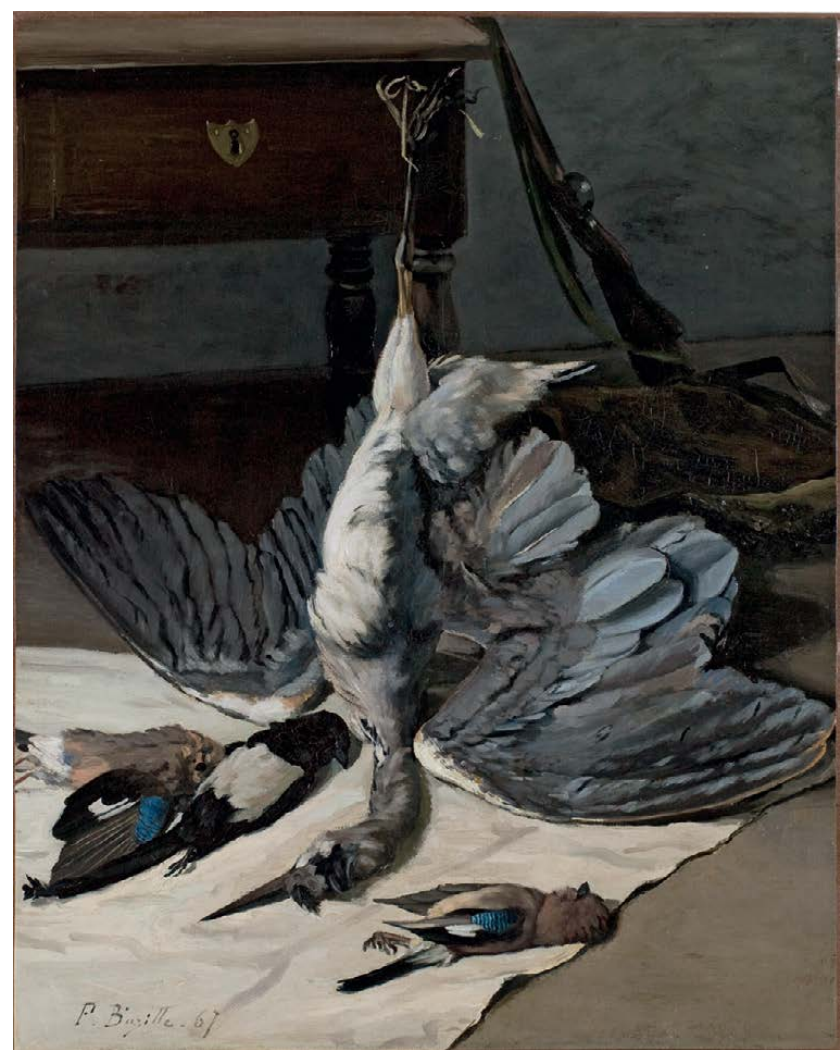

Fig. 6. Frédéric Bazille, Nature morte au héron, 1867, huile sur toile (98 x $78 \mathrm{~cm})$, Montpellier, musée Fabre (inv. 898.5.2). (C) C2RMF/M. de Drée.

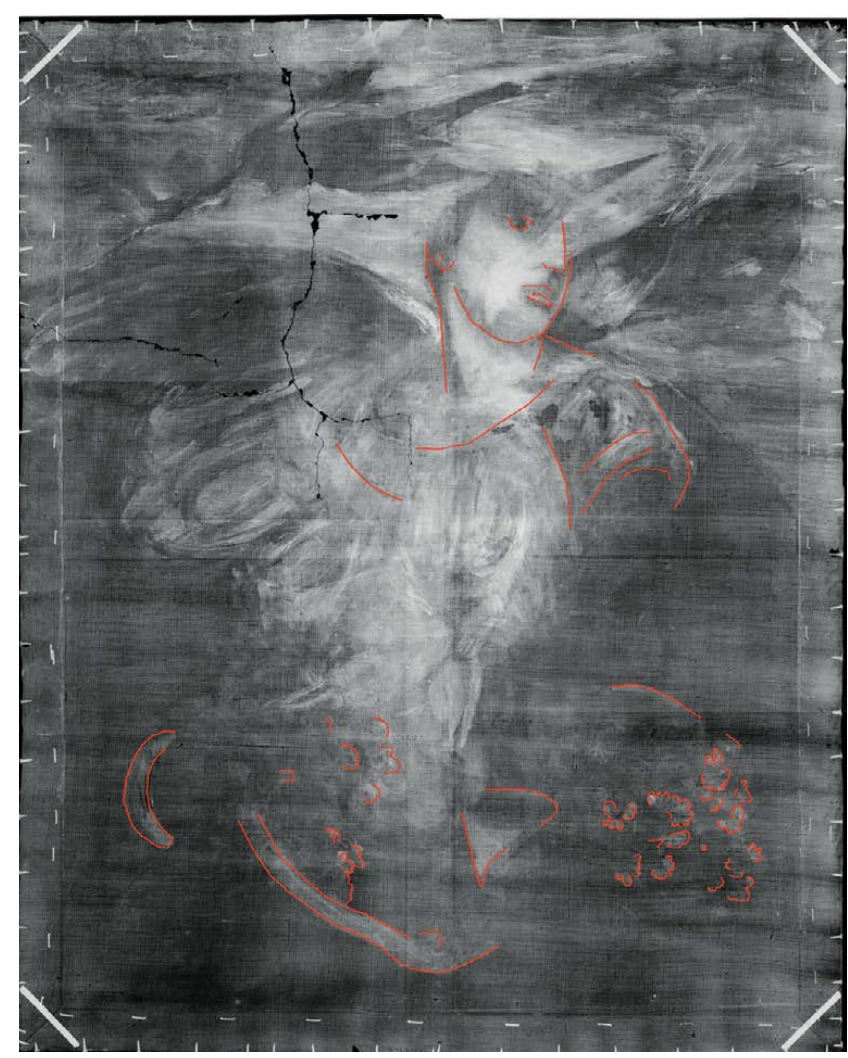

Fig. 7. Nature morte au héron : radiographie présentée tête-bêche, mettant en évidence une Femme aux bouquets. (c) C2RMF/M. de Drée, numérisation et B. Mottin, schéma.

gras dont la présence a gêné le séchage des couches du dessus et entraîné la formation de craquelures prématurées. Bazille a ainsi répété l'erreur qui avait poussé Huguet-Moline à retoucher la première composition.

d'outil à la surface. Après avoir effacé presque entièrement sa première composition, il a repris la figure en diminuant l'ampleur de la robe, en déplaçant les pieds, en tournant le buste et en supprimant un bras, en modifiant la ligne du cou et en détournant peut-être le visage du modèle.

La Robe rose a donc été commencée en 1864, mais a été largement transformée après février 1865. On comprend ainsi pourquoi la mise en page de l'œuvre et sa haute qualité d'exécution diffèrent tant des autres créations datées de 1864, telles que la copie grasse et approximative du Mariage mystique de sainte Catherine de Véronèse (Beaune-la-Rolande, église), ou que l'Étude de nu du musée Fabre, au chromatisme un peu trivial. Aucun document ne permet de dater cette reprise. On proposera d'en situer l'exécution au cours du séjour de l'artiste à Méric durant l'été 1865, guère au-delà, car la peinture et la préparation devaient être encore fraîches pour pouvoir être enlevées. La reprise a toutefois été faite sur un fond trop

\section{Repentirs et toiles de remploi}

La Petite Italienne chanteuse des rues (fig. 4), datée de 1866, est peinte sur une toile lâche, dont la préparation blanche, irrégulière, a été posée par l'artiste. Les contours de la figure sont esquissés avec la même "sauce » brun-rouge que sur La Robe rose, qui est également employée ici en à-plats servant de ton de fond à la composition. Ce ton est clair au premier plan, il est plus sombre à l'emplacement des immeubles où il est laissé visible. La fillette est peinte au-dessus avec des couleurs franches posées en fines couches qui n'ont pas posé de problème de séchage. Les empâtements sont localisés sur les fleurs du chapeau ainsi que sur les immeubles, lesquels sont traités au moyen de petits bâtonnets verticaux dont la couleur 


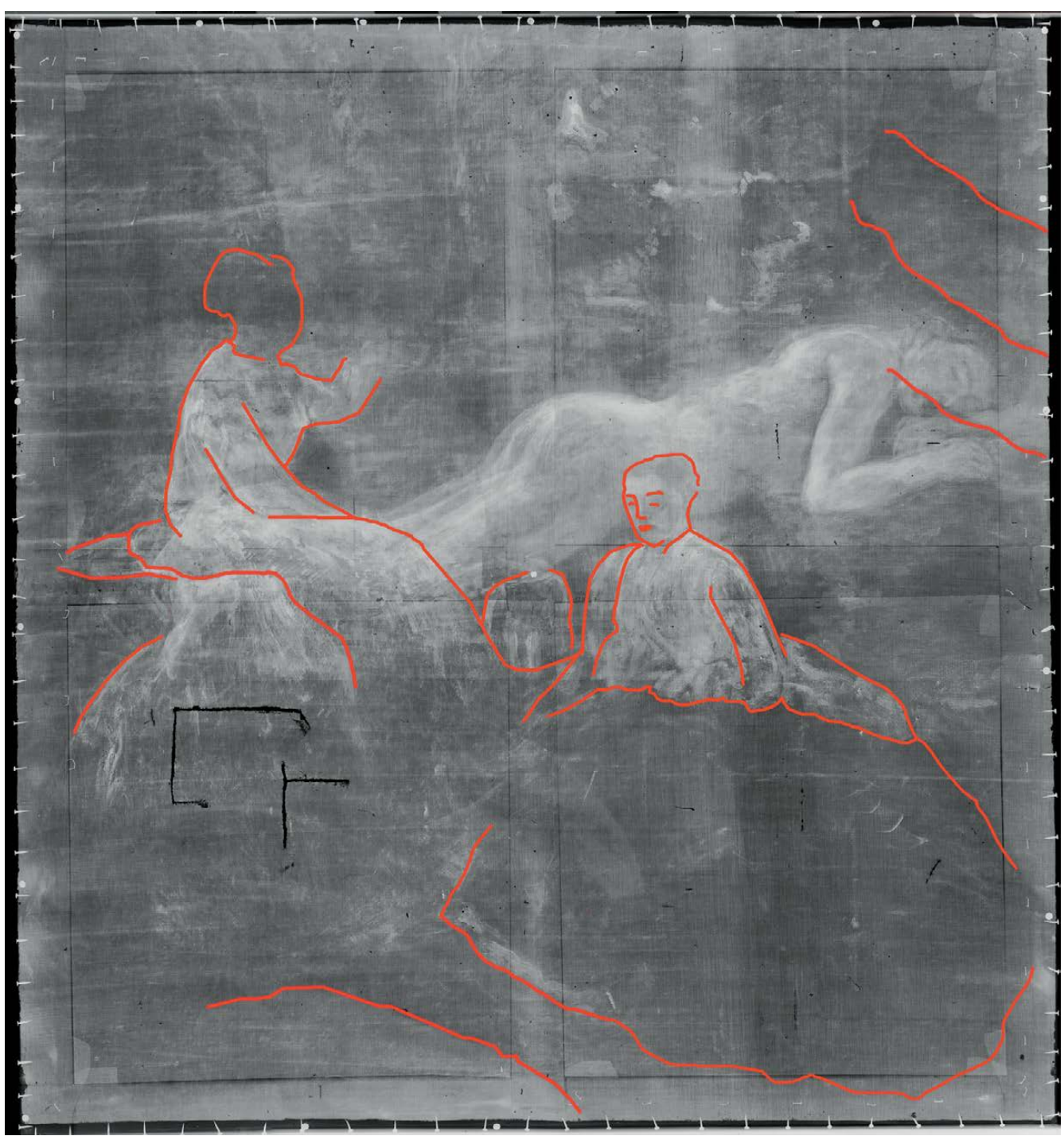

et le relief varient selon la distance, dans une modernité de traitement digne d'un Manet.

Bazille abandonne le ton brun rouge de l'ébauche dans ses œuvres ultérieures, et le remplace par des tons locaux, comme on l'observe au bas du Jeune homme nu (1870) (fig. 8), où une première composition sous-jacente, représentant des femmes assises, est mise en place au moyen de tons bruns, bleus et mauves. La Réunion de famille (1867-1868) est esquissée selon le même procédé, avec des tons variés où les visages sont curieusement préparés par une sous-couche gris bleuté. La radiographie de ce tableau (fig. 5) confirme que la composition était initialement très proche de deux dessins préparatoires, aujourd'hui au musée d'Orsay, et que l'œuvre a été transformée en cours de peinture ${ }^{6}$. La composition était initialement fermée sur la gauche par un seul personnage, l'oncle
Eugène des Hours, au ventre rebondi et portant haut-deforme ; Bazille a ajouté son autoportrait en déplaçant son oncle vers la droite et en le mettant de face. Au centre, Thèrèse des Hours, qui est à présent assise sur une chaise, se tenait debout et dialoguait avec sa mère, Adrienne, assise derrière la table ; on devine le premier emplacement de sa tête dissimulé dans les feuillages. À droite, le groupe était disposé différemment.

Les repentirs en cours de composition sont peu fréquents sur les autres tableaux. On ne mentionnera que le cas du Portrait d'Alphonse Tissié en tenue de cuirassier (vers 1868) dont la tête a été entièrement repeinte sur une tête probablement trop grosse. Cette reprise de composition semble avoir alourdi la toile au tissage trop lâche ( $14 \times 12$ fils au cm), au point de motiver la décision de faire rentoiler l'œuvre en fin d'exécution, en conservant le châssis, mais en dépliant légèrement les bords de la toile. 


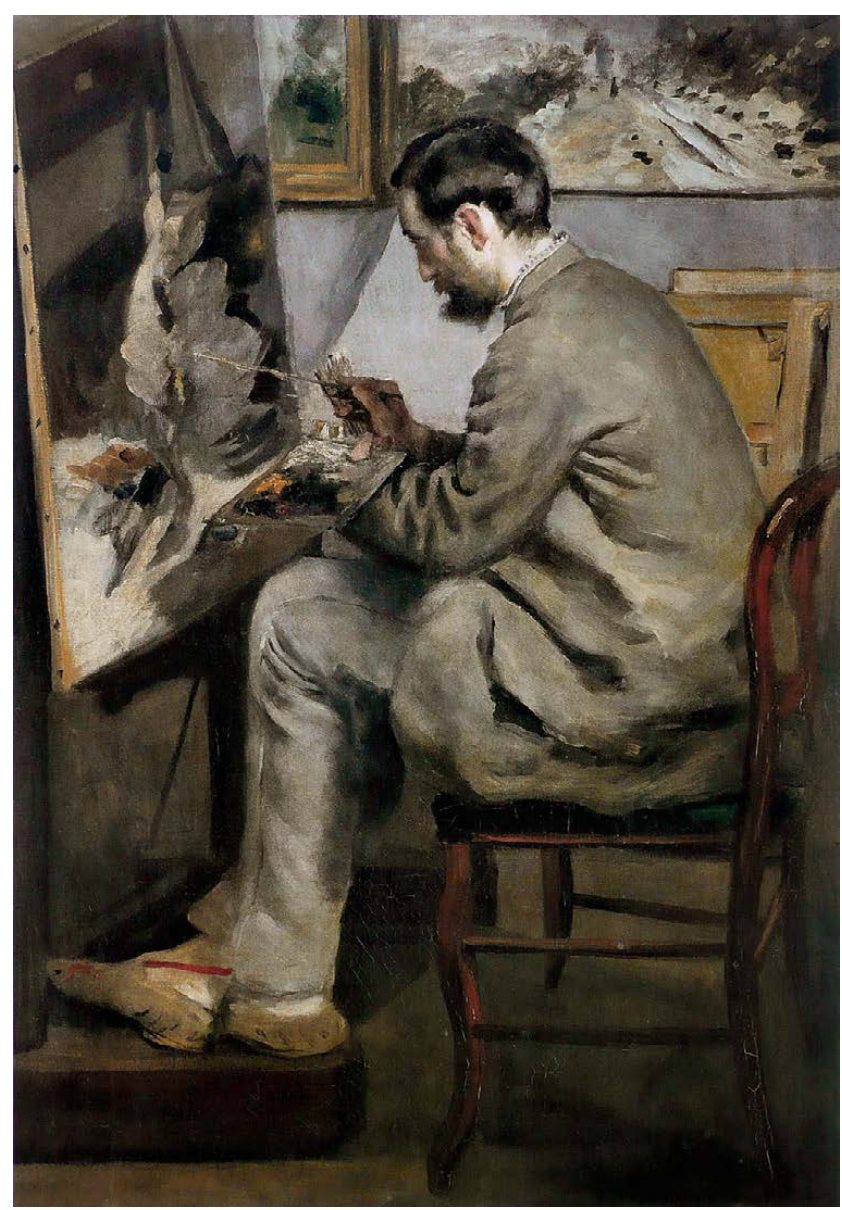

Fig. 9. Auguste Renoir, Bazille peignant le héron, 1867, huile sur toile $(105 \times 73 \mathrm{~cm})$, Paris, musée d'Orsay, en dépôt à Montpellier, musée Fabre (inv. RF 2448). (C) C2RMF/M. de Drée.

Le rentoilage a été réalisé par le marchand Hardy-Alan autour de 1868 car la toile porte le cachet de ce fabricant à l'adresse du 1 rue Childebert, alors que celui-ci déménage au 36 rue du Cherche-Midi en $1869^{7}$. On s'étonne que cette toile inachevée et non accidentée ait fait l'objet d'un rentoilage ; le seul autre exemple connu d'un rentoilage précoce concerne la Scène d'été (1869-1870) que Bazille doit faire rentoiler par Hardy-Alan à cause d'un accident de transport ${ }^{8}$.

Les réutilisations de toiles déjà peintes sont nombreuses. Paul Perrin a montré comment un tableau réputé perdu, $L a$ Jeune fille au piano, refusé au salon de 1865, a pu être retrouvé grâce à la radiographie sous la forme d'une composition sousjacente au Ruth et Booz (1870) de Montpellier ${ }^{9}$. Après le refus de l'œuvre, Bazille a conservé la toile roulée pendant près de cinq ans avant de la retendre sur un châssis légèrement plus petit pour peindre, tête-bêche, une nouvelle composition. La toile originale porte le cachet du fabricant « Hardy-Alan et Mme Picard, Marchands de Couleur fines, 1 rue Childebert à Paris ", marque utilisée entre 1857 et 1868 alors que le Ruth et Booz est peint en 1870 : l'indice confirme, si cela était nécessaire, qu'une toile plus ancienne a été utilisée.

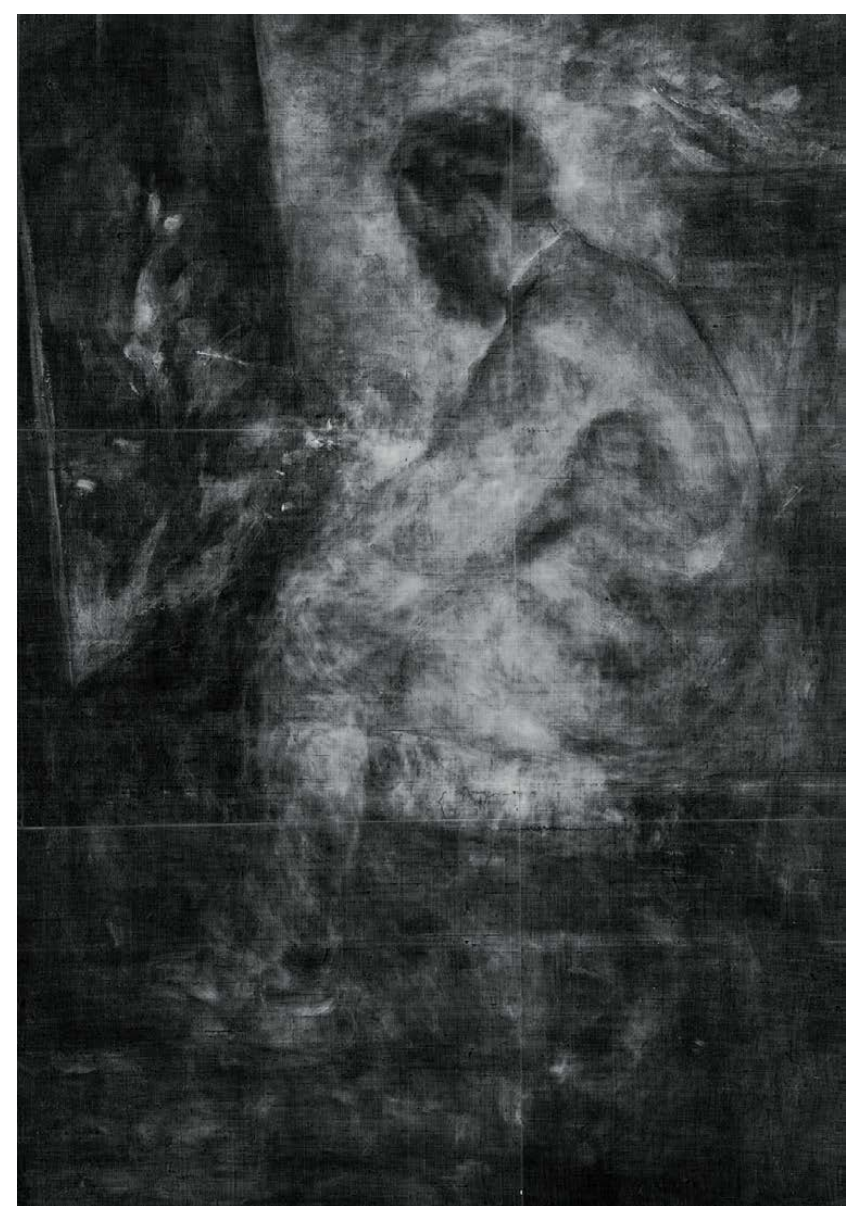

Fig. 10. Auguste Renoir, Bazille peignant le héron, radiographie. (C) C2RMF/M. de Drée.

Les Femmes assises peintes au-dessous du Jeune homme nu (1870) de Montpellier (fig. 8) ne peuvent, quant à elles, constituer une étude préparatoire au Déjeuner sur l'herbe de Claude Monet, contrairement à la proposition de Kermit Champa ${ }^{10}$. La confrontation avec les radiographies du Déjeuner sur l'herbe montre en effet que leur technique est très différente. Le visage de la femme de droite figurant sous le Jeune homme nu est fortement charpenté et construit avec une matière épaisse, qui diffère de la touche fluide de Monet alors qu'elle est identique à celle de la composition peinte au-dessus. Bazille semble donc l'auteur des deux compositions et n'a pas emprunté de toile à Claude Monet.

La Nature morte au héron (fig. 6) est peinte tête-bêche sur un portrait de femme se tenant derrière des fleurs (fig. 7). Paul Perrin ${ }^{11}$ a observé que le portrait est préparé par une Étude de jeune femme, actuellement en mains privées, dont l'attitude est la même et qui porte une chemise à manches courtes identique. Les pots de fleurs et le bouquet sont comparables à une Étude de fleurs (1866) également en collection particulière. Le peintre envisageait donc, dès 1866-1867, de peindre une jeune femme aux fleurs, thème qu'il développera en 1870 avec les deux versions de la Négresse aux pivoines. 


\section{La communauté d'artistes}

L'aspect le plus touchant de la personnalité de Bazille est la facilité avec laquelle il accueille les artistes de son entourage et travaille avec eux. Le musée de Montpellier a la chance d'en posséder une triple illustration avec les deux Nature morte au héron, peintes par Bazille et Sisley, et avec le Portrait de Bazille peignant le héron, exécuté par Renoir au même moment (fig. 9). Sur le tableau de Renoir, la préparation claire est encore visible dans l'angle inférieur droit du tableau que peint Bazille. On remarque que la queue de la pie est parallèle au bec du héron et pointe vers le bas alors qu'elle longe le geai de gauche sur le tableau achevé. L'examen de la radiographie du Héron de Bazille confirme que la queue de la pie a d'abord été peinte comme l'a représentée Renoir, puis a été déplacée. La pie ressemblait donc à celle de Sisley, et si ce dernier n'a pas représenté de geai au premier plan, à la différence de Bazille, la radiographie de son propre tableau montre qu'il en avait réservé l'emplacement, mais a recouvert cette zone d'un ton blanc. Les deux peintres ont donc travaillé ensemble, devant un même motif qu'ils ont légèrement modifié en cours d'exécution.

La radiographie du Portrait de Bazille permet en outre d'observer la technique toute particulière de PierreAuguste Renoir (fig. 10). Ce dernier a suivi une formation de peintre sur porcelaine et se vante d'être resté trop peu de temps chez Gleyre pour avoir été déformé par son métier académique. De fait, Renoir peint au moyen de petites touches fines et brouillées, presque sans matière, qui rappellent la minutie du peintre sur porcelaine. La tête de Bazille devait initialement se détacher sur un mur nu, où elle était comme nimbée de lumière. Elle n'était pas placée devant les deux tableaux fixés au mur, qui ont été peints dans un second temps. On peut émettre l'hypothèse que le tableau de droite, représentant un Paysage de neige de Claude Monet, a pu être peint par Monet lui-même, et non par Renoir, car tous ces peintres travaillent à ce moment-là dans le même atelier. Monet aurait ainsi ajouté sa présence sur cette œuvre d'amitié dont on rappellera que le premier propriétaire en fut Édouard Manet.
Depuis une proposition formulée par Anne Distel ${ }^{12}$, on pense que le fameux Atelier de la rue La Condamine (fig. 11) recouvre une composition sous-jacente peinte par Renoir. La radiographie de ce tableau (fig. 12) permet en effet d'observer une femme nue, de face, assise sur une sellette, les deux bras relevés vers la droite, sujet qui peut être mis en relation avec un grand tableau peint par Renoir en 1867 représentant une Diane chasseresse $^{13}$. La comparaison de cette composition

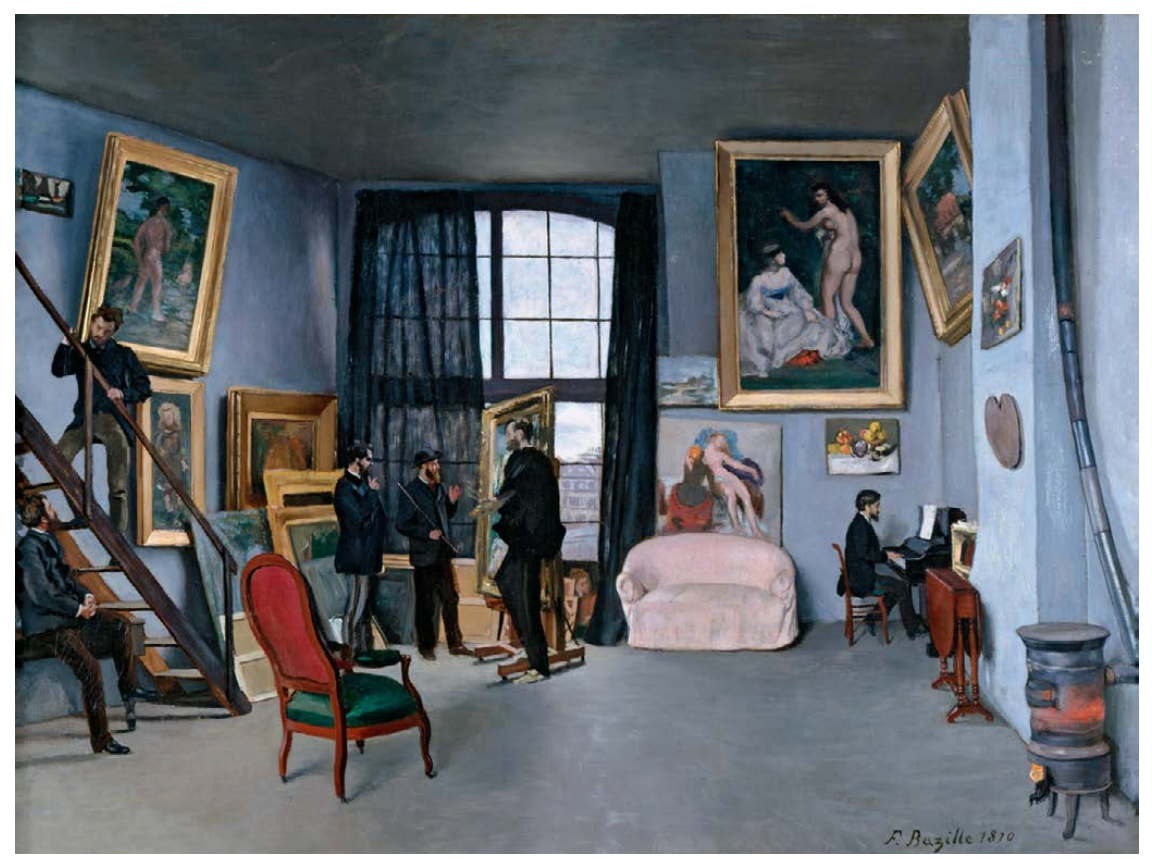

Fig. 11. Frédéric Bazille, Atelier de la rue La Condamine, 1870, huile sur toile $(98 \times 128 \mathrm{~cm})$, Paris, musée d'Orsay (inv. RF 2449). (C C2RMF/J. Marsac.

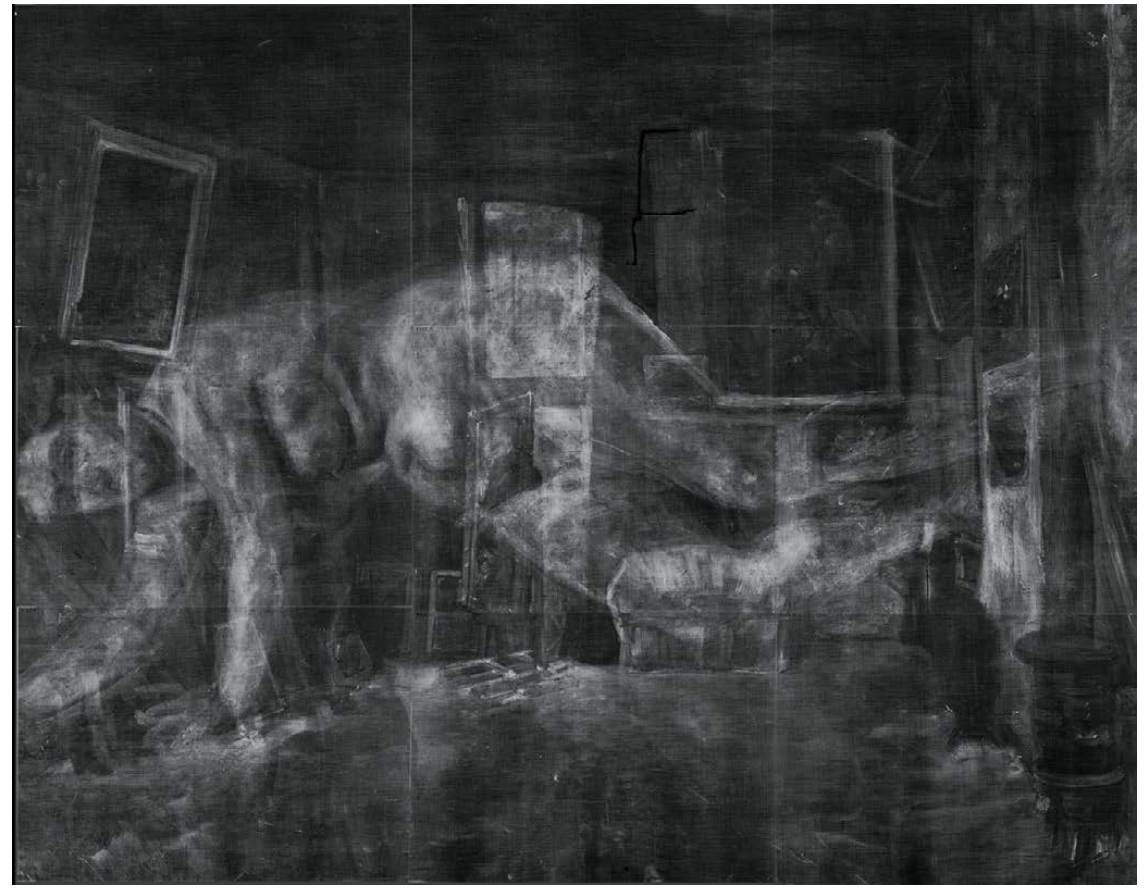

Fig. 12. Atelier de la rue La Condamine: radiographie. (c) C2RMF/J. Marsac. 
sous-jacente avec les radiographies des tableaux de Renoir de la même époque, tels que Bazille peignant le héron (fig. 9), le Garçon au chat (1868) ou le Poirier d'Angleterre (vers 1870) suffit toutefois pour démontrer que Renoir ne peut être l'auteur de l'étude de femme, car la figure est trop charpentée par rapport à la technique du peintre. Il s'agit probablement d'une œuvre de Bazille, peinte en parallèle au tableau de Renoir et d'après le même modèle.

L'Atelier de la rue La Condamine contient d'autres signes de l'amitié puissante qui unissait ce petit cercle. Cette vue d'atelier sublimée, à la perspective invraisemblable et aux proportions surdimensionnées, a d'abord été peinte vide de personnages. Sur la radiographie (fig. 12), Edmond Maître n'est pas au piano, personne ne se tient autour du grand tableau sur chevalet et l'angle inférieur gauche du Pêcheur à l'épervier n'est pas caché par l'homme gravissant l'escalier. Les personnages ont été posés après coup, alors que le tableau était déjà mis en couleurs et avant les finitions. On pense que le portrait du grand Bazille a été peint par Édouard Manet.
Les autres personnages ont pu être peints au fur et à mesure du passage des modèles, ce qui expliquerait leurs disparités de proportions. Il est amusant de noter qu'un personnage inattendu s'est invité en cours d'exécution : il s'agit du beau fauteuil Voltaire qui faisait partie du mobilier du peintre depuis son arrivée à Paris et dont l'emplacement n'est pas réservé sur le premier projet.

En résumé, les études de laboratoire permettent de mieux comprendre le processus d'exécution complexe de La Robe rose et d'en repousser la phase principale d'exécution autour de 1865. Elles révèlent d'importants repentirs, notamment sur la Réunion de Famille, ainsi que la présence de nombreuses compositions sous-jacentes qui semblent toutes de la main de Bazille, et non de Renoir ou de Monet. Elles permettent enfin de mieux comprendre comment fonctionnait la fraternité d'artistes qui donnera naissance à l'impressionnisme, fondée sur un partage d'ateliers et de modèles où chacun a pu intervenir sur les œuvres de ses camarades, mais de façon très limitée.

\section{Notes}

1. Liste des œuvres étudiées.

Paris, musée d'Orsay : La Robe rose (1864-1865), L'Ambulance improvisée (1865), Réunion de famille (1867), Portrait de Renoir (vers 1868-1869), L’Atelier de la rue La Condamine (1869), par Frédéric Bazille ; Jeune garçon au chat, par Auguste Renoir, Fragments du Déjeuner sur l'herbe par Claude Monet.

Montpellier, musée Fabre : Petite Italienne chanteuse des rues (1866), Nature morte au héron (1867), Portrait d'Alphonse Tissié en cuirassier (1868), Jeune homme nu (1870), Ruth et Booz (1870) par Frédéric Bazille ; Nature morte au héron par Alfred Sisley (1867), Portrait de Bazille peignant le héron, par Auguste Renoir (1867).

Aucune ouvre n'a fait l'objet de prélèvement.

2. Frédéric Bazille, Portrait de Thérèse des Hours, assise sur une terrasse, crayon noir sur papier, 47,5 x 30,5 cm, Paris, musée du Louvre, fonds d'arts graphiques du musée d'Orsay (inv. RF 29730 recto).

3. Lettre de Gaston Bazille à son fils, 16 décembre 1864, dans Schulman, 1995, document 96, p. 341.
4. Lettre de Frédéric Bazille à sa mère, février 1865 ?, dans Schulman, 1995, document 101, p. 343.

5. Callen, 1982 (2006).

6. Frédéric Bazille, La Réunion de famille sur la terrasse du château de Méric; crayon noir sur papier, $30,1 \times 30 \mathrm{~cm}$ (inv. RF 5257 recto) et 23,9 x 30,2 cm (inv. RF 5258 recto), Paris, musée d'Orsay, en dépôt au musée du Louvre, département des Arts graphiques.

7. Labreuche, 2011; www.labreuchefournisseurs-artistes-paris.fr.

8. Exp. Montpellier, Paris, Washington, 2016, p. 152.

9. Exp. Montpellier, Paris, Washington, 2016, p. 74-79.

10. Champa, 1973

11. Exp. Montpellier, Paris, Washington, 2016, cat. 34, p. 237.

12. Exp. Paris, Boston, 1985.

13. Pierre-Auguste Renoir, Diane chasseresse, 1867, huile sur toile, 199, 5 x 129,5 cm, Washington, National Gallery of Art (inv. 1963.10.2005).

\section{Bibliographie}

Callen A., 1982, Techniques of the Impressionnists, Orbis, Londres (trad. française, 2006), p. 189.

Champa K., 1973, Studies in Early Impressionism, Yale University Press, New Haven et Londres, p. 86.

Exp. Paris, Boston, 1985, Renoir [Cat. Exp. Paris, Grand Palais, Boston, Museum of fine Arts, 1985-1986], sous la direction de Distel A., Éditions RMN-Grand Palais, Paris, p. 60.

Exp. Montpellier, Paris, Washington, 2016, Frédéric Bazille, la jeunesse de l'impressionnisme [Cat. Exp. Montpellier, musée Fabre, Paris, musée d'Orsay, Washington, National Gallery of Art, 2016-2017], sous la direction de Hilaire M. et Perrin P., Flammarion, Paris.

Labreuche P., 2011, Paris, capitale de la toile à peindre, $X_{V I I I}{ }^{e}-X I X^{e}$ siècle, CTHS-INHA, Paris.

Schulman F., 1995, Frédéric Bazille, 1841-1870, catalogue raisonné, Éditions de l'Amateur/Éditions des catalogues raisonnés, Paris. 